\title{
Prospect theory for continuous distributions: A preference foundation
}

\author{
Amit Kothiyal · Vitalie Spinu • Peter P. Wakker
}

Published online: 28 April 2011

(C) The Author(s) 2011. This article is published with open access at Springerlink.com

\begin{abstract}
Preference foundations give necessary and sufficient conditions for a decision model, stated directly in terms of the empirical primitive: the preference relation. For the most popular descriptive model for decision making under risk and uncertainty today, prospect theory, preference foundations have as yet been provided only for prospects taking finitely many values. In applications, however, prospects often are complex and involve infinitely many values, as in normal and lognormal distributions. This paper provides a preference foundation of prospect theory for such complex prospects. We allow for unbounded utility and only require finite additivity of the underlying probability distributions, leaving the restriction to countably additive distributions optional. As corollaries, we generalize previously obtained preference foundations for special cases of prospect theory (rank-dependent utility and Choquet expected utility) that all required countable additivity. We now obtain genuine generalizations of de Finetti's and Savage's finitely additive setups to unbounded utility.
\end{abstract}

Keywords Prospect theory • Preference foundations .

Continuous distributions $\cdot$ Unbounded utility

JEL Classification $\mathrm{D} 81 \cdot \mathrm{C} 60$

The most popular descriptive theory of decision making under risk and uncertainty today is prospect theory. Its original version was introduced by

\footnotetext{
A. Kothiyal · V. Spinu · P. P. Wakker $(\bowtie)$

Econometric Institute, Erasmus University, P.O. Box 1738,

Rotterdam, 3000 DR, The Netherlands

e-mail: wakker@ese.eur.nl
} 
Kahneman and Tversky (1979), a paper that constituted a breakthrough in decision theory. Up to that point, the common thinking had been that irrational behavior was too chaotic to be modeled, and that models of rational choice were the best descriptive approximation of irrational behavior (Arrow 1951, p. 406). Prospect theory was the first convincing model that at the same time was tractable enough to allow for theoretical analyses and predictive applications, and was also able to model irrationalities commonly found in empirical choices.

Original prospect theory has some drawbacks. First, there are some theoretical problems in the way it implemented nonadditive probabilities. Second, it deals only with risk (known probabilities). Third, within risk it deals only with a limited set of prospects (only two nonzero outcomes). ${ }^{1}$ Quiggin (1982) discovered the solution to the first problem for risk. Schmeidler (1989, first version 1982) independently discovered that same solution and, importantly, extended it to uncertainty (unknown probabilities), jointly with Gilboa (1987), which resolves the second problem. Quiggin could handle any finite set of outcomes, not just the case of two nonzero outcomes, and Schmeidler could handle all bounded prospects, which attenuates the third problem. A limitation of their solution was, however, that they could not handle reference dependence and loss aversion, important empirical components of prospect theory, so that their solution applies only to gains or losses separately. It cannot handle mixed cases with both gains and losses. Tversky and Kahneman (1992) incorporated reference dependence and loss aversion in the models of Quiggin and GilboaSchmeidler, leading to new prospect theory, which will be called prospect theory henceforth. ${ }^{2}$ Thus they obtained the first theory that combines theoretical soundness with empirical realism.

The absence of any preference foundation for original prospect theory could have served as a signal of something being wrong. ${ }^{3}$ Preference foundations state conditions, directly in terms of preferences, that are necessary and sufficient for some decision model, such as prospect theory, to hold. Because preferences are directly observable, preference foundations identify the empirical meaning of a model. They show how to directly verify or falsify a model empirically, and how to defend or criticize it normatively. If the conditions in the preference foundation are natural then the theoretical soundness of the model has been established.

\footnotetext{
${ }^{1}$ Extensions to more nonzero outcomes, proposed in the literature, all have serious problems (Wakker 2010, Appendix 9.8).

${ }^{2}$ The new version is, as we see it, an advance relative to the original version, as expressed in the title of the 1992 paper. Hence we use the convenient term prospect theory for the new theory rather than for the original one, in agreement with the terminology in Wakker, Thaler, and Tversky (1997) for instance.

${ }^{3}$ Kahneman and Tversky (1979, Appendix), aware of the importance of preference foundations, tried to provide one as good as possible, but they only obtained a partial foundation and could not identify the full empirical meaning of their model.
} 
Historical examples illustrate the importance of preference foundations. The Bayesian approach to economics and statistics became popular through famous preference foundations by de Finetti (1931), von Neumann and Morgenstern (1944), and Savage (1954). The multiple priors model, introduced by Wald (1950) and extensively discussed as early as in Luce and Raiffa (1957, Ch. 13), only became popular when Gilboa and Schmeidler (1989) provided a preference foundation.

In retrospect it is clear why a preference foundation (with natural conditions) could never have been found for original prospect theory: the functional of this theory is not theoretically sound. The other theories discussed above did receive a preference foundation. Most of those preference foundations only concerned simple prospects, being prospects that take only finitely many values; Schmeidler (1989) could handle all bounded prospects. The preference foundations of Quiggin (1982) and Schmeidler (1989) were extended to continuous and possibly unbounded prospects by Wakker (1993).

Tversky and Kahneman (1992) did not define or analyze their new theory for continuous distributions. Such a definition can readily be given (Wakker and Tversky 1993, Eq. 6.1 and Section 8.4; Davies and Satchell 2003; Wakker 2010, Eqs. 9.7.1 and 12.8.1). This paper will present a preference foundation of the new version of prospect theory for continuous distributions. It is desirable to establish such a foundation because continuous distributions are widely used in applications. For example, stock prices are usually assumed to be lognormally distributed in finance (Hull 2006). Investment decisions deal with continua of investment options and, then, with continua of possible returns. Economic growth models consider continuous paths over time with continuous risks (Becker and Boyd 1997). Health economics typically deals with continuous outcome scales such as quality of life, considering continuous probability distributions over those scales and, further, profiles that are continuous over time (Drummond et al. 1987). References applying prospect theory with nonlinear probability weighting to continuous distributions, often lognormal and, hence, unbounded, include Barberis and Huang (2008), Barberis and Xiong (2009), Bernard and Ghossoub (2009), Carlier and Dana (2011), He and Zhou (2010), Henderson (2010), Kanbur et al. (2008), Spalt (2010), and Sumalee et al. (2009).

Another difference between the modern version of prospect theory and its original version is that until recently no one could even define the latter for continuous distributions in any plausible manner. Only recently Rieger and Wang (2008) succeeded in proposing such an extension. They showed that, basically, only the derivative $w^{\prime}(0)$ of the weighting function $w$ at $p=0$ is empirically relevant for continuous distributions. In our interpretation, this is unsatisfactory. Thus Rieger and Wang revealed further limitations of original prospect theory. Their approach can be extended to all theories using transformations of probabilities of separate outcomes (Allais 1979; Edwards 1962; Viscusi 1989).

In what follows, Section 1 deals with decision under risk. It defines prospect theory for continuous distributions and presents the preference foundation for 
this case. Our result generalizes Wakker (1993, Corollary 4.5) by incorporating reference dependence and loss aversion. It is also structurally more general. As a corollary, we generalize Wakker's extension of Quiggin's (1982) rankdependent utility from countably additive to the more general finitely additive prospects.

Section 2 provides extensions to uncertainty, which is modeled through states of nature and events for which no probabilities are available (ambiguity). This result, in combination with the aforementioned extension to finite additivity, implies that we obtain genuine generalizations of de Finetti (1931, 1972) and Savage (1954), allowing for unbounded utility. An additional feature of our approach is that the only richness required is that all simple (finitely valued) prospects are available, plus truncations of the prospects considered. Other than that, our results can be applied to any domain of prospects. It thus serves as justification of using prospect theory in many applications, including applications that consider only normal, or lognormal, distributions and their truncations. Section 3 concludes. Appendix A shows how measure-theoretic structure can be introduced, and Appendices B and C give proofs. Appendix C introduces a method for embedding decision under risk into decision under uncertainty that generalizes Wakker's (1993, Section 4.2) technique.

\section{Risk}

Structural definitions $X$ is an outcome set. It is often taken to be the set $\mathbb{R}$ of reals, designating monetary outcomes. Everything that follows remains valid for more general outcome sets, such as commodity bundles or health states. We will assume for the utility function defined later that its range is a nonpoint interval, which will imply that the outcome set is a continuum and cannot be finite. One outcome, the reference outcome, denoted $r$, will play a special role in what follows. A common case is $r=0$ for monetary outcomes, designating the status quo.

A probability distribution $P$ on $X$ maps the subsets of $X$ to $[0,1]$, with $P(\emptyset)=0, P(X)=1$, and

$$
A \cap B=\emptyset \Rightarrow P(A \cup B)=P(A)+P(B) \text { (additivity). }
$$

Sometimes a stronger property, countable additivity, is useful:

If a countable sequence of events $A_{j}$ are mutually disjoint then $P\left(\cup_{j=1}^{\infty} A_{j}\right)=$ $\sum_{j=1}^{\infty} P\left(A_{j}\right)$.

$\mathcal{P}$ is a subset of the set of all probability distributions over $X$. Its elements are called prospects. Our analysis will be flexible regarding the domain $(\mathcal{P})$ of prospects. Our analysis holds with no modification if we restrict attention to countably additive prospects. It also holds if we add measure theoretic structure (Appendix A).

$\mathcal{P}^{s}$ denotes the set of simple prospects, i.e. the prospects that assign probability 1 to a finite set of outcomes. A simple prospect can be denoted ( $p_{1}$ : $\left.x_{1}, \ldots, p_{n}: x_{n}\right)$ with the obvious meaning. $\succcurlyeq$ is a preference relation over 
$\mathcal{P} . \succ, \preccurlyeq, \prec$, and $\sim$ are defined as usual. Any degenerate prospect $(1: \alpha)$, assigning probability 1 to an outcome $\alpha$, is identified with that outcome. Preferences over prospects thus generate preferences over outcomes. Outcomes strictly preferred to $r$ are gains, and outcomes strictly less preferred than $r$ are losses. Outcomes indifferent to $r$ are neutral. For prospect $P$, outcome $\alpha$ is a certainty equivalent $(C E)$ if $\alpha \sim P$. A function $V$ represents $\succcurlyeq$ if $V: \mathcal{P} \rightarrow \mathbb{R}$ and

$$
P \succcurlyeq Q \Leftrightarrow V(P) \geq V(Q) .
$$

Defining prospect theory A function $w$ is a (probability) weighting function if it maps $[0,1]$ to itself, satisfies $w(0)=0$ and $w(1)=1$, and is nondecreasing. Under prospect theory, we use a different weighting function for gains than for losses.

Definition 1 Prospect theory (PT) holds if there exist weighting functions $w^{+}$and $w^{-}$, and a utility function $U: X \rightarrow \mathbb{R}$ with $U(r)=0$, such that the evaluation

$$
\begin{aligned}
P \rightarrow P T(P)= & \int_{\mathbb{R}_{+}} w^{+}(P\{\alpha \in X: U(\alpha) \geq \tau\}) d \tau \\
& -\int_{\mathbb{R}_{-}} w^{-}(P(\{\alpha \in X: U(\alpha) \leq \tau\}) d \tau
\end{aligned}
$$

is well defined and real-valued for all prospects $P \in \mathcal{P}$, and represents preferences on $\mathcal{P}$. Then $P T(P)$ is the prospect theory value of $P$.

Tversky and Kahneman (1992) only considered simple prospects, for which the integral in our Eq. 1 coincides with their definition of PT. Equation 1 agrees with Quiggin's (1982) rank-dependent utility for gains separately, and also for losses separately. It may deviate when both gains and losses are present. We have maintained the classical symbol $U$ and the classical term utility, instead of the symbol $v$ and the term value function that Kahneman and Tversky used. For losses, our function $U$ implicitly includes loss aversion; i.e., it is the loss aversion parameter times the value function of prospect theory. This is why we do not explicitly write the loss aversion parameter in this paper. Wakker (2010) called $U$ the overall utility function.

Preference conditions for prospect theory If PT holds and, more generally, if a representing function exists, then $\succcurlyeq$ is a weak order. That is, then $\succcurlyeq$ is complete $(P \succcurlyeq Q$ or $Q \succcurlyeq P$ for all $P, Q \in \mathcal{P})$ and transitive. For the extension of PT from simple to bounded prospects, we use the following two preference conditions.

(i) Certainty equivalence, or $C E$ equivalence, holds if for each prospect $P \in \mathcal{P}$ there exists a CE.

(ii) (Weak) stochastic dominance holds if, for all $P, Q \in \mathcal{P}$, we have $P \succcurlyeq Q$ whenever: $P\{\beta \in X: \beta \preccurlyeq \alpha\} \leq Q\{\beta \in X: \beta \preccurlyeq \alpha\}\}$ for all $\alpha \in X$. 
We next consider the extension to prospects with unbounded utility (of their outcomes) that, however, still have a finite and well-defined PT value. For this purpose we use a truncation-continuity condition, introduced by Wakker (1993). The condition imposes restrictions on the preference relation in combination with the preference domain $\mathcal{P}$. It will rule out all unbounded prospects that have infinite or undefined PT values, and will do so entirely in terms of preferences.

For prospect $P$ and outcome $\mu, P^{\wedge \mu}$, the above truncation of $P$ at $\mu$, is equal to $P$ on $\{\alpha \in X: \alpha \preccurlyeq \mu\} \backslash\{\mu\}$, and assigns all remaining probability $P\{\mu\}+$ $P\{\alpha \in X: \alpha \succ \mu\}$ to outcome $\mu$. For outcome $v, P_{\vee v}$, the below truncation of $P$ at $v$, is equal to $P$ on $\{\alpha \in X: \alpha \succcurlyeq v\} \backslash\{v\}$, and assigns all remaining probability $P\{v\}+P\{\alpha \in X: \alpha \prec v\}$ to outcome $v$.

$P$ is truncation-robust if for all outcomes $\alpha$ the following holds:

$$
\begin{aligned}
& P \succ \alpha \Longrightarrow \exists \mu \in X: P^{\wedge \mu} \in \mathcal{P}, P^{\wedge \mu} \succ \alpha ; \\
& P \prec \alpha \Longrightarrow \exists v \in X: P_{\vee} \in \mathcal{P}, P_{\vee} \prec \alpha .
\end{aligned}
$$

All bounded prospects (defined formally in Appendix B), which includes all simple prospects, trivially are truncation-robust. Truncation-continuity holds if all prospects $P \in \mathcal{P}$ are truncation-robust. We will assume the following richness on $\mathcal{F}$ : Truncation-closedness holds if for every $P \in \mathcal{P}$ and $\alpha \in X$, we have $P^{\wedge \alpha} \in \mathcal{P}$ and $P_{\vee \alpha} \in \mathcal{P}$.

Our main theorem for risk We will not relate the following theorem to one particular preference foundation of PT. We will state it in general, so that it can be used as an add-on to every preference foundation of PT for simple prospects that has a continuum of utility. This includes the common cases with real-valued outcomes or commodity bundles as outcomes and with continuous utility. Preference foundations for PT have so far mostly been studied for uncertainty. Chateauneuf and Wakker (1999) gave a preference foundation for risk with a continuum of utility, but only for simple prospects. Theorem 2 can be combined with their result to include continuous distributions.

Theorem 2 Assume that PT holds on $\mathcal{P}^{s}$, with utility function $U$ and weighting functions $w^{+}$and $w^{-}$. Assume the following richness:

(1) $U(X)$ is an interval that contains 0 in its interior.

(2) We have: $\mathcal{P}^{s} \subset \mathcal{P}$, certainty equivalence, and truncation-closedness.

Then PT holds on all of $\mathcal{P}$ with respect to the same $U, w^{+}$, and $w^{-}$, with the PT value of all prospects in $\mathcal{P}$ well defined and finite, if and only if the following conditions hold on $\mathcal{P}$ :

(i) Weak ordering;

(ii) Stochastic dominance;

(iii) Truncation-continuity. 
Wakker (1993, Corollary 4.5) did not use the proof technique that we use in Appendix C (constructing the state space $S$ as a high-dimensional product space) and, hence, provided his extension of rank-dependent utility to unbounded prospects only for countably additive prospects. We can now extend it to finitely additive prospects. Another difference between Wakker's (1993) analysis and ours is that he used a step equivalence assumption instead of our simpler certainty equivalence assumption. If the utility range is a continuum, as in Wakker's Corollary 4.5 and in our paper, then equivalence of the two conditions follows elementarily. A third difference is that Wakker (1993) assumed a condition called weak truncation-closedness, instead of our truncationclosedness, throughout his paper. Our results could be generalized to Wakker's condition. That condition is, however, more complex, and we nowadays prefer accessibility to mathematical generality.

Corollary 3 Under truncation-closedness, Corollary 4.5 of Wakker $(1993)^{4}$ also holds without the restriction of countable additivity.

\section{Uncertainty}

Structural definitions $X$ is again an outcome set, with $r$ the reference outcome. Now no probabilities are given for uncertainties, but uncertainties are modelled through states (of nature). $S$ denotes the state space. Exactly one state is true, the others are not true, and the decision maker does not know for sure which state is true. Events are subsets of the state space. An event is true if it contains the true state of nature. $\emptyset$, the vacuous event, surely is not true, and $S$, the universal event, surely is. The results in this paper all hold true both for finite and for infinite state spaces $S$. For finite state spaces they, however, do not contribute anything new, with all extra conditions vacuously satisfied. Only for infinite state spaces do our results bring novelty.

We consider a subset $\mathcal{F}$ of the set of all functions from $S$ to $X$. Its elements are called prospects. ${ }^{5}$ We assume that $\mathcal{F}$ contains the set $\mathcal{F}^{s}$ of all simple functions from $S$ to $X$, taking only finitely many outcomes. A simple prospect can be denoted $\left(E_{1}: x_{1}, \ldots, E_{n}: x_{n}\right)$. Here it is implicitly understood that $E_{1}, \ldots, E_{n}$ are events that partition the state space. The prospect assigns outcome $x_{j}$ to every state in $E_{j}$. Using the same letter $\mathrm{s}$ to indicate states of nature and, as a superscript, to indicate simple prospects will not raise confusion.

$\succcurlyeq$ is a preference relation over $\mathcal{F}$, with $\succ, \preccurlyeq, \prec$, and $\sim$ as usual. Any degenerate prospect $(S: \alpha)$, assigning outcome $\alpha$ to each $s \in S$, is identified with

\footnotetext{
${ }^{4}$ This corollary is the analog of Theorem 2 for Quiggin's rank-dependent utility instead of prospect theory.

${ }^{5}$ To distinguish the prospects defined in this section from the prospects defined before for decision under risk, the term event-contingent prospect can be used here. We can then call the prospects for risk probability-contingent.
} 
that outcome $\alpha$. Preferences over prospects thus, again, generate preferences over outcomes. Gains, losses, neutral outcomes, certainty equivalents, and representing functions are defined as before.

Defining prospect theory A function $W$ is a weighting function if it maps events to $[0,1]$, and satisfies $W(\emptyset)=0, W(S)=1$, and $A \supset B \Rightarrow W(A) \geq$ $W(B)$ (set monotonicity). Weighting functions need not satisfy additivity. Probability measures concern the special case of weighting functions that do satisfy additivity.

Definition 4 Prospect theory $(P T)$ holds if there exist weighting functions $W^{+}$and $W^{-}$, and a utility function $U: X \rightarrow \mathbb{R}$ with $U(r)=0$, such that the evaluation

$$
f \rightarrow P T(f)=\int_{\mathbb{R}_{+}} W^{+}\{s \in S: U(f(s)) \geq \tau\} d \tau-\int_{\mathbb{R}_{-}} W^{-}\{s \in S: U(f(s)) \leq \tau\} d \tau
$$

is well defined and real-valued for all prospects $f \in \mathcal{F}$, and represents preferences on $\mathcal{F}$.

Preference conditions for prospect theory Certainty equivalence (CE equivalence) holds if for every prospect in $\mathcal{F}$ there exists a $\mathrm{CE}$. The following condition adapts stochastic dominance from risk to uncertainty. Pointwise monotonicity holds if, for all $f, g \in \mathcal{F}$, we have $f \succcurlyeq g$ whenever $f(s) \succcurlyeq g(s)$ for all states $s$.

For prospect $f$ and outcome $\mu, f^{\wedge \mu}$, the above truncation of $f$ at $\mu$, is defined by

$$
\begin{aligned}
& f^{\wedge \mu}(s)=\mu \text { if } f(s) \succ \mu ; \\
& f^{\wedge \mu}(s)=f(s) \text { if } f(s) \preccurlyeq \mu .
\end{aligned}
$$

The below truncation of $f$ at $v, f_{v v}$, is defined by

$$
\begin{aligned}
& f_{v v}(s)=v \text { if } f(s) \prec v ; \\
& f_{V v}(s)=f(s) \text { if } f(s) \succcurlyeq v .
\end{aligned}
$$

Prospect $f \in \mathcal{F}$ is truncation-robust if for all outcomes $\alpha$ the following holds:

$$
\begin{aligned}
& f \succ \alpha \Longrightarrow \exists \mu \in X: f^{\wedge \mu} \in \mathcal{F}, f^{\wedge \mu} \succ \alpha ; \\
& f \prec \alpha \Longrightarrow \exists v \in X: f_{\vee v} \in \mathcal{F}, f_{\vee v} \prec \alpha .
\end{aligned}
$$

Truncation-continuity holds if all prospects $f \in \mathcal{F}$ are truncation-robust. We will assume the following richness on $\mathcal{F}$ : truncation-closedness holds if for every $f \in \mathcal{F}$ and $\alpha \in X$, we have $f^{\wedge \alpha} \in \mathcal{F}$ and $f_{\vee \alpha} \in \mathcal{F}$. As in the case of risk we will not relate the following theorem to one particular preference 
foundation of PT. We will state it in general, so that it can be used as an addon to every preference foundation of PT existing today. Thus, we assume that a PT representation has been obtained for the simple prospects, and that the utility range is a continuum. These conditions are satisfied for all PT preference foundations that exist today. References are given after the following theorem.

Theorem 5 Assume that PT holds on $\mathcal{F}^{s}$, with utility function $U$ and weighting functions $W^{+}$and $W^{-}$. Assume the following richness:

(1) $U(X)$ is an interval that contains 0 in its interior.

(2) We have: $\mathcal{F}^{s} \subset \mathcal{F}$, certainty equivalence, and truncation-closedness.

Then PT holds on all of $\mathcal{F}$ with respect to the same $U, W^{+}$, and $W^{-}$, with the $P T$ value of all prospect in $\mathcal{F}$ well defined and finite, if and only if the following conditions hold on $\mathcal{F}$ :

(i) Weak ordering;

(ii) Pointwise monotonicity;

(iii) Truncation-continuity.

Theorem 5 can be combined with any presently existing preference foundation of PT (all obtained only for simple prospects) to provide a complete preference foundation of PT including nonsimple prospects. The presently existing preference foundations include Köbberling and Wakker (2003, Theorem 12), Luce and Fishburn (1991, Theorem 5 and its corollary), Tversky and Kahneman (1992, Theorem 2), Wakker and Tversky (1993, Theorem 6.3), and Wakker (2010, Theorem 12.3.5). It may seem that certainty equivalence is implied by the $P T$ representation, given that $U(X)$ is an interval, in which case the certainty equivalence condition could have been added to the necessary and sufficient conditions in the theorem. In virtually all cases of interest this is correct. There are, however, exceptional examples in which this is not so (Example 10 in Appendix B), because of which the condition has been stated as a richness restriction.

\section{Conclusion}

Prospect theory had as yet only received preference foundations for simple prospects (taking finitely many values). Applications in finance, health, and other domains often deal with complex situations with continua of possible outcomes. This paper has given a preference foundation for such cases, i.e. preference conditions that provide the critical demarcation for validity of the theory in such cases. Thus this paper supports applications of prospect theory to situations of complex uncertainties. 


\section{Appendix A: Measure-theory for risk and uncertainty}

It is often useful, when working with measures and weighting functions, to add measure-theoretic structure. This appendix explains how this can be done. Nothing in the analyses of this paper in the main text needs to be modified if such structure, as defined later, is added. We will prove our main results in following appendices assuming such structure. Let us point out, prior to defining this structure through algebras $\mathcal{D}$ and $\mathcal{A}$ below, that absence of measuretheoretic structure concerns, per definition, the special case where the algebras $\mathcal{D}$ and $\mathcal{A}$ contain all subsets of $X$ and $S$, respectively. Then all measuretheoretic restrictions are trivially satisfied and can be ignored. Thus the proofs in following appendices are also valid under absence of measure-theoretic structure.

An algebra on a set (the universal set) is a nonempty collection of subsets of the universal set that is closed under complementation and finite unions. It follows that it contains $\emptyset$, the universal set, and is also closed under finite intersections. A trivial example is the algebra of all subsets. Another example, for the universal set $\mathbb{R}$, is the collection of all finite unions of intervals. A third example is the usual Borel sigma-algebra on $\mathbb{R}$, which is defined as the smallest collection closed under complementation and countable unions that contains all intervals.

To add measure-theoretic structure to our analysis, we, throughout, endow $X$ with an algebra $\mathcal{D}$ on $X$. We assume that $\mathcal{D}$ contains all preference intervals. A preference interval is a subset $Y$ of $X$ such that $\beta \in Y$ whenever $\alpha \preccurlyeq \beta \preccurlyeq \gamma$ for some $\alpha, \gamma \in Y$. It means that $\mathcal{D}$ contains all $U^{-1}(I)$ for intervals $I$ under PT, both for risk and for uncertainty. For simplicity, we assume that $\mathcal{D}$ contains all singletons. ${ }^{6}$ Because of this assumption, we need not distinguish between simple prospects and step prospects.

Because we allow algebras rather than the more common sigma algebras, some extra care must be taken in the proofs. For example, sums, differences, and mixtures of measurable prospects now need not be measurable (Wakker 1993, Example 1.2). But maximums and minimums of two measurable prospects, as in truncations, are. We will often use the latter implicitly in the proofs that follow.

Decision under risk Now prospects are defined only on elements of $\mathcal{D}$. That is, they specify only for elements $D$ of $\mathcal{D}$ what the probability is that the outcome

\footnotetext{
${ }^{6}$ This can always be arranged by replacing all outcomes by their preference indifference classes. In view of pointwise dominance for uncertainty and stochastic dominance for risk, replacing outcomes in a prospect by other equivalent outcomes does not affect the preference value of the prospect, so that preferences over prospects are well defined if only indifference classes of outcomes are specified. Because indifference classes are preference intervals and, hence, are contained in $\mathcal{D}$, we can modify $\mathcal{D}$ accordingly.
} 
of the prospect will be contained in $D$. Nothing more needs to be adapted in the analysis. Measure-theoretic structure is needed, for instance, if we want to study atomless (e.g. continuous) countably additive prospects. The reason is that an atomless prospect defined on the collection of all subsets of a continuum such as $X$ cannot be countably additive (Banach and Kuratowski 1929; Ulam 1930). Its domain therefore has to be restricted.

Weak stochastic dominance implies, for $X=\mathbb{R}$, that only the distribution function $(\alpha \rightarrow P((-\infty, \alpha])$ is relevant for the preference value of a prospect $P$. In other words, two prospects that have the same distribution function are equally preferred. The latter requirement is automatically satisfied for countably additive $P$ on the Borel sets because then the distribution function completely determines $P$. It is restrictive for finitely additive prospects, i.e. prospects that need not be countably additive.

Decision under uncertainty We assume an algebra $\mathcal{A}$ on $S$ in addition to the algebra $\mathcal{D}$ on $X$. Now events are only the elements of $\mathcal{A}$. We assume measurability of prospects: For every $f \in \mathcal{F}$ and $D \in \mathcal{D}, f^{-1}(D) \in \mathcal{A}$. This condition is satisfied for all prospects if $\mathcal{A}$ contains all subsets of $S$.

\section{Appendix B: Proof of Theorem 5}

As explained in Appendix A, the following proof is valid both if the measuretheoretic structure of Appendix A is added and if it is not. A useful point to note is that the PT functional (Definition 4), is a sum of two Choquet integrals, because of which it inherits many properties of the Choquet integral.

We first show that PT implies the preference conditions. Weak ordering is obvious, and pointwise monotonicity follows from substitution. For truncation continuity, assume $f \succ \alpha$. Then the integral in Eq. 4 exceeds $U(\alpha)$. There must be a $\mu^{\prime}>0$ so large that taking the left integral in Eq. 4 over $\left[0, \mu^{\prime}\right]$ instead of taking it over $\mathbb{R}^{+}$already gives a result in Eq. 1 that exceeds $U(\alpha){ }^{7}$ If $U(X)$ is bounded above, then we can take $\mu^{\prime}$ smaller than $\sup (U(X))$ but close enough. We define $\mu=U^{-1}\left(\mu^{\prime}\right) . P^{\wedge \mu} \succ \alpha$ follows. The case $f \prec \alpha$ is treated similarly. Truncation continuity follows.

We next assume the preference conditions in Theorem 5, and derive PT. We first state a special case in the following lemma and then derive the general case.

Lemma 6 Assume that the outcome space $X$ is an interval in the real line containing $r=0$ in its interior and that utility $U$ is the identity $(U(\alpha)=\alpha)$. For this special case, Theorem 5 holds.

\footnotetext{
${ }^{7}$ This can be seen most easily by viewing the left integral as a regular integral of the function $W^{+}\{s \in S: U(f(s)) \geq \tau\}$. (The integral over $\mathbb{R}^{+}$is per definition the limit of the integrals over $\left[0, \mu^{\prime}\right]$.) It can also be seen by viewing the left integral as a Choquet integral.
} 
Proof Now $r=0$. The proof will comprise some lemmas, whose proofs are ended with $Q E D$. For each $f$ in $\mathcal{F}$, define $V(f)=U(\alpha)=\alpha$ with $\alpha$ a CE of $f$. By $\mathrm{CE}$ equivalence, such a CE always exists. Under the assumption of linear utility as made here, the $\mathrm{CE}$ is unique because different outcomes are never indifferent. Obviously, $V(f)$ is real-valued for each $f$. By weak ordering, $V$ represents preference. On $\mathcal{F}^{s}, V$ agrees with PT. We show that $V$ also agrees with the PT functional on the rest of $\mathcal{F}$. $V$ satisfies pointwise monotonicity in the sense that $f(s) \succcurlyeq g(s) \Rightarrow V(f) \geq V(g)$. We call $f$ bounded above if there exists an outcome $\alpha$ such that $\forall s \in S: \alpha \succcurlyeq f(s) .{ }^{8}$ We call $f$ bounded below if there exists an outcome $\beta$ such that $\forall s \in S: f(s) \succcurlyeq \beta$. We call $f$ bounded if it is both bounded above and bounded below.

Lemma 7 If $f$ is bounded below then $V(f) \geq P T(f)$. If $f$ is bounded above then $V(f) \leq P T(f)$. This implies in either case that $P T(f)$ is finite.

Proof We only consider the case where $f$ is bounded below. This implies that $P T(f)$ is well defined and is either finite or $+\infty$. We define a sequence of outcomes $\alpha_{n}$ tending to the upper bound of $X$. Then $f^{\wedge \alpha_{n}}$ is bounded for each positive integer $n$. It is well known that, for each $n$, we can define a sequence of simple prospects $\left(f_{n, k}^{s}\right)_{k=1}^{\infty}$ approximating $f^{\wedge \alpha_{n}}$ from below (Wakker 1993, Lemma 1.4). For the PT functional, being a sum of two Choquet integrals, we get

$$
\lim _{n \rightarrow \infty} \lim _{k \rightarrow \infty} P T\left(f_{n, k}^{s}\right)=\lim _{n \rightarrow \infty} P T\left(f^{\wedge \alpha_{n}}\right)=P T(f) .
$$

For each $\mathrm{n}$ and $\mathrm{k}, V(f) \geq V\left(f_{n, k}^{s}\right)=P T\left(f_{n, k}^{s}\right)$ by pointwise monotonicity of $V$. Hence $V(f) \geq P T(f)$. In view of finiteness of $V, P T(f)$ cannot be $+\infty$ and must be finite too.

$Q E D$

Corollary 8 The functional $P T$ is well defined and finite for all $f$.

Proof Take any prospect $f$, and outcome $\alpha$. By Lemma $7, f^{\wedge \alpha}$ has a finite $P T$ integral. This implies that the negative part of the $P T(f)$ integral, which is the PT integral of $f^{\wedge 0}$ and which differs by no more than $|\alpha|$ from the $P T$ integral of $f^{\wedge \alpha}$, is finite too. Similarly, $f_{\vee \alpha}$ and the positive part of the $P T(f)$ integral are finite too. $P T(f)$, the sum of its positive and negative parts, is finite too.

$Q E D$

Lemma $9 V(f)=P T(f)$ for all $f$.

Proof For contradiction, assume $V(f)>P T(f)$. The case $V(f)<P T(f)$ is similar. $V(f)=C E(f)$. Because $P T(f)$ is smaller, there must exist outcomes

\footnotetext{
${ }^{8}$ This condition is slightly more restrictive than the common condition of boundedness if $U(X)$ is bounded above but does not contain its supremum.
} 
smaller than $V(f)$, and we can find an outcome $\alpha$ with $V(f)=C E(f)>\alpha>$ $P T(f)$. There exists an outcome $\mu$ with $f^{\wedge \mu} \succ \alpha>P T(f) \geq P T\left(f^{\wedge \mu}\right)$. Then $V\left(f^{\wedge \mu}\right)>\alpha>P T\left(f^{\wedge \mu}\right)$. This contradicts Lemma 7.

$Q E D$

Lemma 6 has been proved.

The PT representation for Theorem 5 follows readily from Lemma 6 by replacing all outcomes $\alpha \in X$ by $U(\alpha)$ and transforming the model accordingly. This transformation does not affect anything relevant. Details are as follows. For each $\alpha, \alpha^{\prime}=U(\alpha)$, and for each $f, f^{\prime}$ is defined by $f^{\prime}(s)=U(f(s))$. $\mathcal{F}^{\prime}=\left\{f^{\prime}: f \in \mathcal{F}\right\}$, and $f^{\prime} \succcurlyeq^{\prime} g^{\prime}$ if and only if $f \succcurlyeq g$. $\succcurlyeq^{\prime}$ satisfies all conditions in Lemma 6. The resulting $P T^{\prime}$ representation for $\succcurlyeq^{\prime}$ generates a $P T$ representation for $\succcurlyeq$. The proof of Theorem 5 is complete.

The following example shows why certainty equivalence is not implied by the representation in our theorems, so that it had to be added as a richness condition. The example is pathological in considering what are known as completely finitely additive probability measures. It can be seen that these are the only cases where certainty equivalence is not implied by the representation.

Example 10 Let $S=\mathbb{N}$, with $\mathcal{A}$ containing all subsets of $S$. Let $W^{+}=W^{-}=P$ be a finitely additive measure on $S$ assigning probability 0 to every finite subset of $S$. Such finitely additive probabilities exist. Because $P$ is additive, this is the special case where $P T$ is expected utility. Let $X=[-1,1)$ with $U$ the identity. Let $f(s)=1-1 / s$. Then $P(f(s)>1-1 / j)=1$ for all $j \in \mathbb{I}$, and $P T(f)=1$. It is strictly preferred to every outcome in $X$, and there exists no certainty equivalent of $f$.

\section{Appendix C: Proof of Theorem 2 and Corollary 3, and an example}

As explained in Appendix A, the following proof is valid both if the measuretheoretic structure of Appendix A is added and if it is not. We first consider Theorem 2. It easily follows that PT implies the preference conditions: Weak ordering is obvious, and stochastic dominance follows from substitution. The proof of truncation continuity is very similar to the proof of Theorem 5 and is omitted.

We next assume the preference conditions in Theorem 2, and derive PT. We derive this from Theorem 5 by developing a homomorphism between risk and uncertainty. This homomorphism is of interest on its own, because it shows how theorems for decision under risk with finitely additive probabilities can be derived from theorems for decision under uncertainty with state spaces.

Definition of the homomorphism We define a state space so that each probability-contingent prospect $P$ corresponds with an event-contingent prospect. A convenient construction for countably additive probability measures, used by Wakker (1993, Section 4.2), is through generalized inverses 
of distribution functions. We, however, also allow for finite additivity, where distribution functions are more complex and do not fully characterize the probability measure. We, hence, define an alternative homomorphism that can handle finite additivity. It is in the spirit of Kolmogorov's (1950) extension theorem for countable additivity.

We define $S$ as the product space $X^{\mathcal{P}}$ with a separate dimension for each $P \in \mathcal{P}$. The algebra $\mathcal{A}$ on $S$ contains all "cylinder" sets $Y \times X^{(\mathcal{P}-\{P\})}$ where $Y \in$ $\mathcal{D}$ and $Y$ concerns dimension $P$, their finite intersections ("cubes"), and their finite unions. That is, events (elements of $\mathcal{A}$ ) specify what happens for finitely many dimensions, and leave all other dimensions unrestricted. We endow this algebra with a finitely additive probability $\mathcal{Q} . \mathcal{Q}$ assigns to each "marginal" $Y \times X^{(\mathcal{P}-\{P\})}$ (with $Y \in \mathcal{D}$ referring to dimension $P$ ) the probability $P(Y) . \mathcal{Q}$ is the product measure of the corresponding marginal probabilities (i.e., they are taken as independent).

Every $\mathcal{A}-\mathcal{D}$ measurable function $f^{\prime}$ from $S$ to $X$ generates a probability distribution $f$ over $X$ in the usual way. We define as $\mathcal{F}$ the set of all such measurable functions that generate a probability distribution that is contained in $\mathcal{P}$. We define $\succcurlyeq^{\prime}$ on $\mathcal{F}$ by $f^{\prime} \succcurlyeq^{\prime} g^{\prime}$ if $f \succcurlyeq g$ where $f$ and $g$ are generated by $f^{\prime}$ and $g^{\prime}$. We trivially have $f^{\prime} \sim g^{\prime}$ whenever $f=g$, a condition known as probabilistic sophistication (Wakker 2010, Section 10.1).

Completing the proof using the homomorphism We verify that $\succcurlyeq^{\prime}$ satisfies all preference conditions of Theorem 5. Measurability, $\mathcal{F}^{s} \subset \mathcal{F}$, and CE equivalence are obvious. For truncation-closedness, note that each $f \in \mathcal{P}$ is generated by at least one element of $\mathcal{F}$ : the projection on the dimension of $S$ corresponding with $f$. Also note that truncations of $f^{\prime} \in \mathcal{F}$ correspond with truncations of the generated $f \in \mathcal{P}$ in the natural manner. For example, it can be seen that $f^{\prime \wedge \mu}$ generates $f^{\wedge \mu}$. Now truncation-closedness of $\succcurlyeq$ implies it for $\succcurlyeq^{\prime}$.

We next turn to the necessary and sufficient preference conditions. Weak ordering holds obviously, and pointwise monotonicity directly follows from stochastic dominance. So all that remains to be proved is truncation-continuity of $\succcurlyeq^{\prime}$.

Assume $f^{\prime} \succ^{\prime} \alpha$. Then for the corresponding $f$ we have $f^{\wedge \mu} \succ \alpha$ for some $\mu$. $f^{\prime \wedge \mu} \in \mathcal{F}$ by definition of $\mathcal{F}$, and $f^{\prime \wedge \mu} \succ^{\prime} \alpha$ by definition of $\succcurlyeq^{\prime}$. We can similarly show that $f^{\prime} \prec^{\prime} \alpha$ implies $f_{v v}^{\prime} \prec^{\prime} \alpha$ for some $v$. Truncation-continuity holds. All conditions of Theorem 5 are satisfied, and we obtain a $P T$ representation of $\succcurlyeq^{\prime}$.

To obtain a $P T$ representation of $\succcurlyeq$, for each $p \in[0,1]$ we take an event $E \in \mathcal{A}$ with $\mathcal{Q}(E)=p$. Such an event $E$ always exists because every $P=(p$ : $\alpha, 1-p: \beta)$ is contained in $\mathcal{P}$ and the event $\{\alpha\} \times X^{(\mathcal{P}-\{P\})}$ has probability $p$. We define $w^{+}(p)=W^{+}(E)$ and $w^{-}(p)=W^{-}(E)$. This definition does not depend on the particular event $E$ chosen: Any two events $A, B$ with the same $\mathcal{Q}$ probability $p$ have $\left(A: \alpha, A^{c}: \beta\right) \sim\left(B: \alpha, B^{c}: \beta\right)$ for all $\alpha \succ \beta \succcurlyeq r$ and also for all $\alpha \prec \beta \preccurlyeq r$ so that they have the same $W^{+}$value and the same $W^{-}$value. We have $W^{+}(E)=w^{+}(\mathcal{Q}(E))$ and $W^{-}(E)=w^{-}(\mathcal{Q}(E))$. Now the 
$P T$ representation on $\mathcal{F}$ in Eq. 4 generates that in Eq. 1 on $\mathcal{P}$. The proof of

Theorem 2 is complete.

As to the proof of Corollary 3 , the above proof can with no complication be adapted to the case where there are only gains.

Open Access This article is distributed under the terms of the Creative Commons Attribution Noncommercial License which permits any noncommercial use, distribution, and reproduction in any medium, provided the original author(s) and source are credited.

\section{References}

Allais, M. (1979). The so-called Allais paradox and rational decisions under uncertainty. In M. Allais \& O. Hagen (Eds.), Expected utility hypotheses and the Allais paradox (pp. 437-681). Dordrecht, The Netherlands: Reidel.

Arrow, K. J. (1951). Alternative approaches to the theory of choice in risk-taking situations. Econometrica, 19, 404-437.

Banach, S., \& Kuratowski, K. (1929). Sur une généralisation du problème de la mesure. Fundamentà Mathematicae, 14, 127-131.

Barberis, N., \& Huang, M. (2008). Stocks as lotteries: The implications of probability weighting for security prices. American Economic Review, 98, 2066-2100.

Barberis, N., \& Xiong, W. (2009). What drives the disposition effect? An analysis of a longstanding preference- based explanation. The Journal of Finance, 64, 751-784.

Becker, R. A., \& Boyd III, J. H. (1997). Capital theory; equilibrium analysis and recursive utility. Oxford, UK: Blackwell.

Bernard, C., \& Ghossoub, M. (2009). Static portfolio choice under cumulative prospect theory. Mathematics and Financial Economics, 2, 277-306.

Carlier, G., \& Dana, R.-A. (2011). Optimal demand for contingent claims when agents have law invariant utilities. Mathematical Finance, 21, 169-201.

Chateauneuf, A., \& Wakker, P. P. (1999). An axiomatization of cumulative prospect theory for decision under risk. Journal of Risk and Uncertainty, 18, 137-145.

Davies, G. B., \& Satchell, S. E. (2003). Continuous cumulative prospect theory and individual asset allocation. UK: University of Cambridge.

de Finetti, B. (1931). Sul significato soggettivo della probabilità. Fundamentà Mathematicae, 17, 298-329. Translated into English as "On the subjective meaning of probability". In P. Monari \& D. Cocchi (Eds., 1993) Probabilità e induzione (pp. 291-321). Bologna: Clueb.

de Finetti, B. (1972). Probability, induction and statistics. New York: Wiley.

Drummond, M. F., Stoddart, G. L., \& Torrance, G. W. (1987). Methods for the economic evaluation of health care programmes. Oxford: Oxford University Press.

Edwards, W. (1962). Subjective probabilities inferred from decisions. Psychological Review, 69, $109-135$.

Gilboa, I. (1987). Expected utility with purely subjective non-additive probabilities. Journal of Mathematical Economics, 16, 65-88.

Gilboa, I., \& Schmeidler, D. (1989). Maxmin expected utility with a non-unique prior. Journal of Mathematical Economics, 18, 141-153.

He, X. D., \& Zhou, X. Y. (2010). Portfolio choice under cumulative prospect theory: An analytical treatment. Oxford, UK: Nomura Centre for Mathematical Finance, The University of Oxford.

Henderson, V. (2010). Prospect theory, partial liquidation and the disposition effect. UK: OxfordMan Institute of Quantitative Finance, The University of Oxford.

Hull, J. C. (2006). Options, futures, and other derivatives: Solutions manual. Prentice-Hall, NJ: Englewood Cliffs.

Kahneman, D., \& Tversky, A. (1979). Prospect theory: An analysis of decision under risk. Econometrica, 47, 263-291.

Kanbur, R., Pirttilä, J., \& Tuomala, M. (2008). Moral hazard, income taxation and prospect theory. Scandinavian Journal of Economics, 110, 321-337. 
Köbberling, V., \& Wakker, P. P. (2003). Preference foundations for nonexpected utility: A generalized and simplified technique. Mathematics of Operations Research, 28, 395-423.

Kolmogorov, A. N. (1950). Foundations of the theory of probability. New York: Chelsea.

Luce, R. D., \& Fishburn, P. C. (1991). Rank- and sign-dependent linear utility models for finite first-order gambles. Journal of Risk and Uncertainty, 4, 29-59.

Luce, R. D., \& Raiffa, H. (1957). Games and decisions. New York: Wiley.

Quiggin, J. (1982). A theory of anticipated utility. Journal of Economic Behaviour and Organization, 3, 323-343.

Rieger, M. O., \& Wang, M. (2008). Prospect theory for continuous distributions. Journal of Risk and Uncertainty, 36, 83-102.

Savage, L. J. (1954). The Foundations of statistics (2nd ed., 1972, Dover Publications, New York). New York: Wiley.

Schmeidler, D. (1989). Subjective probability and expected utility without additivity. Econometrica, 57, 571-587.

Spalt, O. G. (2010). Small chances and large gains: Why riskier firms grant more employee stock options. The Netherlands: Dept. of Finance, Tilburg University.

Sumalee, A., Connors, R. D., Luathep, P., Lam, W. H. K., Wong, S. C., \& Lo, H. K. (2009). Network equilibrium under cumulative prospect theory and endogenous stochastic demand and supply. In W. H. K. Lam, S. C. Wong, \& H. K. Lo (Eds.), Transportation and traffic theory 2009 (pp. 19-38). Berlin: Springer.

Tversky, A., \& Kahneman, D. (1992). Advances in prospect theory: Cumulative representation of uncertainty. Journal of Risk and Uncertainty, 5, 297-323.

Ulam, S. (1930). Zur masstheorie in der allgemeinen mengenlehre. Fundamentà Mathematicae, 16, $140-150$.

Viscusi, W. K. (1989). Prospective reference theory: Toward an explanation of the paradoxes. Journal of Risk and Uncertainty, 2, 235-264.

von Neumann, J., \& Morgenstern, O. (1944, 1947, 1953). Theory of games and economic behavior. Princeton, NJ: Princeton University Press.

Wakker, P. P. (1993). Unbounded utility for Savage's "foundations of statistics," and other models. Mathematics of Operations Research, 18, 446-485.

Wakker, P. P. (2010). Prospect theory: For risk and ambiguity. Cambridge, UK: Cambridge University Press.

Wakker, P. P., \& Tversky, A. (1993). An axiomatization of cumulative prospect theory. Journal of Risk and Uncertainty, 7, 147-176.

Wakker, P. P., Thaler, R. H., \& Tversky, A. (1997). Probabilistic insurance. Journal of Risk and Uncertainty, 15, 7-28.

Wald, A. (1950). Statistical decision functions. New York: Wiley. 